\title{
Welcome to Bloods-An International Open Access Journal
}

\author{
Antonino Carbone
}

Professor of Pathology, Former Chairman of Department of Pathology, Centro di Riferimento Oncologico (CRO), National Cancer Institute, Via Franco Gallini 2, 33081 Aviano, Italy; acarbone@cro.it or antonino.carbone@asfo.sanita.fvg.it

Received: 20 February 2020; Accepted: 20 February 2020; Published: 21 February 2020

Hematologists, hematopathologists, cellular biologists, immunologists, and molecular biologists are invited to contribute to Bloods, a new international multidisciplinary journal for clinicians and experimental researchers of neoplastic and non-neoplastic blood diseases.

Clinical data regarding diagnosis and management are primarily addressed to clinical specialists, while advances in pathogenesis, molecular epidemiology, genetics, immunogenetics, and immunotherapy about neoplastic and non-neoplastic blood diseases are primarily addressed to biologists, epidemiologists, pharmacologists, and immunologists.

The journal will be divided into several sections, which would help the management of the journal and to have better quality control of the manuscripts. The Journal will contain several sections by type of disease and by type of approach. Then, the Editorial Board will include expert professionals responsible for the various sections, together with hematologists.

\section{Aims and Scope}

The aim of this journal is to provide a platform for studies from pathogenesis to diagnosis and personalized management through in vivo genomic and immunologic research.

\section{Sections of the Journal}

This Journal will include four provisional sections: (1) Non Neoplastic Blood Disorders, (2) Myeloid Leukemia, (3) Lymphoid Leukemia, and (4) Lymphomas. Subsections or points of view will focus on molecular epidemiology, immunobiology, immunogenetics, genomics, immunotherapy, transplantation, target therapy, basket trials, and confirmatory research studies.

\section{The Journal will Consider for Publication}

- Opinion on new discoveries and editorial (from the Editor).

- Review (from the Editorial Board Members; also some invited reviews from other top experts): A comprehensive review with a minimum of 4000 words, containing at least one figure and/or table. The review should provide concise and precise updates on the latest (in the recent five years) progress made in a given area of research.

- Original article and systematic review or meta-analyses in research.

- Brief report.

- Perspective.

- Communication.

\section{Keywords (Aims)}

Non neoplastic blood disorders; myeloid leukemias; lymphoid leukemias; acute leukemias; chronic leukemias; lymphomas; Hodgkin lymphomas; not Hodgkin lymphomas; virus-associated lymphomas; 
HIV-associated lymphomas; immunodeficiency-associated lymphomas; molecular epidemiology; immunobiology; immunogenetics; genomics; immunotherapy; transplantation, target therapy; basket trials; confirmatory research studies.

Conflicts of Interest: The author declares no conflicts of interest.

(C) 2020 by the author. Licensee MDPI, Basel, Switzerland. This article is an open access article distributed under the terms and conditions of the Creative Commons Attribution (CC BY) license (http://creativecommons.org/licenses/by/4.0/). 